\title{
Muskarin-Syndrom nach Pilzverzehr
}

_Eine 85-jährige Frau stellte sich mit Erbrechen, massiven Diarrhöen und Tachykardie bei Exsikkose zwei Stunden

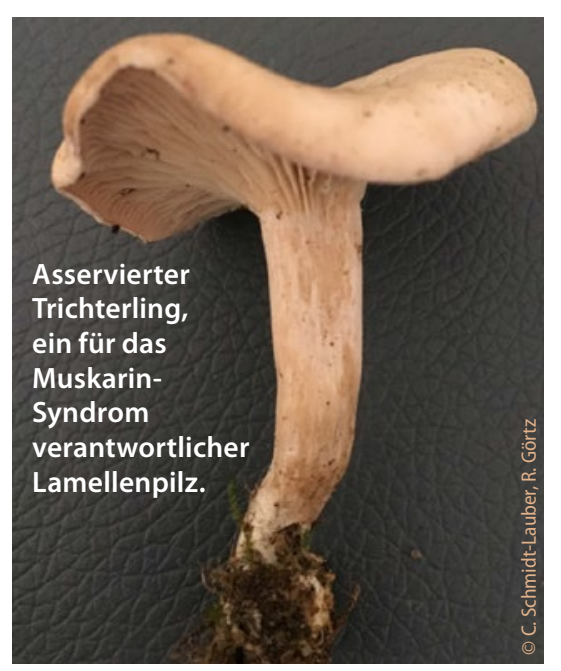

nach Verzehr selbstgesammelter Pilze in der Notaufnahme vor. Neben den Diarrhöen zeigte die Patientin weitere cholinerge Symptome wie Kaltschweißigkeit, Schüttelfrost, Miosis und Verschwommensehen, Hypersalivation mit Schaum vor dem Mund sowie einen Bronchospasmus mit Tachypnoe. Laborchemisch fand sich eine Laktatämie.

Unter dem Verdacht eines MuskarinSyndroms nach Pilzvergiftung erfolgten die Gabe von Atropin, Sauerstoff und niedrig dosiertem Midazolam sowie eine Volumensubstitution. Hierunter kam es innerhalb von Minuten zu einer deutlichen Besserung der Symptomatik. Im Tagesverlauf war die Patientin bereits wieder beschwerdefrei.

Bei der Begutachtung der asservierten Pilze fand sich ein Trichterling (sie- he Abb.). Trichterlinge können Muscarin enthalten, welches ein cholinerges Syndrom auslöst. In diesem Herbst kommen gehäuft gastrointestinale Pilzsyndrome mit kurzer Latenz nach Ingestion von Pilzen vor, bei cholinergen Symptomen muss an ein Muskarin-Syndrom gedacht werden. Bei verzögertem Auftreten von Beschwerden (> 6 Stunden nach Ingestion) sollte eine Amatoxinvergiftung (Knollenblätterpilz) nicht übersehen werden.

\footnotetext{
$\rightarrow$ Dr. med. Christian Schmidt-Lauber PD Dr. med. Rüdiger Stephan Görtz Internistische Notaufnahme der Medizinischen Kliniken 1-5 Universitätsklinikum Erlangen Ulmenweg 18, D-91054 Erlangen E-Mail: ruediger.goertz@uk-erlangen.de
}

\section{7 wichtige Fragen bei Verdacht auf Pilzvergiftung}

Bei Verdacht auf Pilzvergiftung ist die Anamnese von besonderer Bedeutung. Folgende Fragen sollten Sie stellen:

1 Traten die Symptome mit kurzer $(<6$ h) oder langer $(>6 \mathrm{~h})$ Latenzzeit auf?

2 Welche Pilze wurden (vermeintlich) gesammelt? Röhren- oder Lamellenpilze etc.? Pilzgröße, Hutform, Hutfarbe, Lamellenfarbe, Stiel, Knolle, Ring am Stiel, Fundort beschreiben lassen.

3 Wie wurden die Pilze zubereitet und wie lange wurden sie erhitzt? Wie frisch waren sie?

4 Menge der verzehrten Pilze? Handelt es sich um ein Mischpilzgericht?

5 Wurde Alkohol zu der Mahlzeit getrunken?
6 Haben mehrere Leute von der Mahlzeit gegessen? Wenn ja, haben diese auch Symptome?

7 Gibt es Pilzreste (Speisereste, Putzreste, Erbrochenes)? Diese sollten immer asserviert werden.

Über einen Anruf in einem Giftinformationszentrum (Ortsvorwahl-19240) sollte der Kontakt zu einem qualifizierten Pilzsachverständigen in der Umgebung hergestellt werden.

Die Asservierung von Blut und Urin (optimales diagnostisches Fenster 6-36 $\mathrm{h}$ nach der Pilzmahlzeit) ist aus klinischen und forensischen Gründen ratsam.

Vergiftungen mit einer kurzen Latenzzeit sind meist nicht lebensbedroh- lich. Die Therapie richtet sich nach dem Ausmaß der gastrointestinalen Symptomatik sowie der Notwendigkeit der Flüssigkeits- und Elektrolytsubstitution.

Bei einer längeren Latenzzeit und schwerwiegender Symptomatik sollte frühzeitig eine Klinikeinweisung erfolgen. Lässt sich eine Amatoxinvergiftung (Knollenblätterpilzvergiftung!) nicht ausschließen (typische Pilzbeschreibung: weiß- oder grünhütiger Pilz mit weißen Lamellen), sollte schon bei Verdacht eine Silibinintherapie eingeleitet werden.

- red

- Romanek Ket al.: Pilzvergiftungen: Symptome, Diagnostik und Therapie, Notfall Rettungsmed 2016;19:301-314 